\section{Keynote Abstracts}

\section{K-01 AGRICULTURAL EXPOSURES AND HEALTH OUTCOMES}

Laura Beane Freeman. US National Cancer Institute, Division of Cancer Epidemiology \& Genetics, Occupational and Environmental Epidemiology Branch

\subsection{6/OEM-2019-EPI.1}

Pesticides, including herbicides, insecticides, fungicides, fumigants and rodenticides, represent a diverse set of chemicals. They provide important benefits in public health, food production and aesthetics. However, unlike most other chemicals, pesticides are designed to impact living systems. Consequently there has long been a concern about the consequences on both human health and the environment. Pesticide use increased dramatically in the last half of the 20th century, with use continuing to increase in low and middle income countries, reflecting changing agricultural practices. Over 1 billion people are estimated to be exposed to pesticides occupationally through production, mixing, application and other tasks, particularly in the agricultural sector. But, these exposures can also experienced by the general population through various pathways, including drift and contamination of water and food supplies. Not surprisingly, given their diverse properties, various pesticides have been linked to a number of adverse health outcomes, including cancer, respiratory and neurologic disease and reproductive outcomes. With hundreds of pesticide active ingredients and changing practices over time, exposure assessment for etiologic research is a challenge, particularly for chronic diseases with potentially long latency. The ubiquity of the exposure and the concern about health risks by the general population make it imperative to study these important chemicals.

\section{K-02 THE EVOLUTION OF OCCUPATIONAL EPIDEMIOLOGY}

Neil Pearce. Director of the Centre for Global Non-communicable Disease, London School of Hygiene and Tropical Medicine

\subsection{6/OEM-2019-EPI.2}

In this talk, I review the evolution of occupational epidemiology over the last 40 years. Methodologically, the field is almost unrecognizable compared to what was 'standard practice' 40 years ago. Methodological changes include the use of new study design and statistical methods, such as counterfactual theory, directed acyclic graphs (DAGs), IPW, g-estimation, g-computation, multiple imputation for missing values, sensitivity analysis, and bootstrapping. Biomarkers and various molecular and omics measures are increasingly used for exposure assessment, and exploration of mechanisms. The exposures and outcomes under study have also evolved, e.g. with increased consideration of psychosocial factors, work organisation, musculoskeletal problems, mental health and neurological disease. Despite all of these changes, many of the fundamentals of occupational epidemiology remain the same. The discovery of new causes of occupational disease continues to be lead by astute observers (including astute clinicians and astute workers), rather than by 'bigdata' or 'omics' methods. The strategy for investigation of possible occupational causes of disease continues to require a variety of study designs and approaches, including 'descriptive' studies, and triangulation across study designs and populations (albeit while utilising new molecular biology and statistical techniques). The causal assessment of occupational exposures and their health effects continues to require a wide variety of types of evidence in humans and animals, as well as mechanistic evidence. Forty years later, the Bradford-Hill considerations have been augmented but not been replaced, and the IARC 'rules' for combining various types of evidence remain the state-of-the-art. Reports of the death of 'traditional epidemiology' (and its replacement by 'modern epidemiology' and 'causal inference' methods) have been exaggerated.

\section{K-03 A GLOBAL DISEASE BUt A LOCAL PHENOMENOM}

Jackie Benschop. School of Veterinary Science, Massey University, New Zealand

\subsection{6/OEM-2019-EPI.3}

Leptospirosis is a globally important multi-host, multi-pathogen zoonosis with over 1 million cases and 60,000 deaths annually. Humans are infected through contact with urine from infected mammals including wildlife, rodents, farmed species and pets. Despite extensive nationwide intervention measures, leptospirosis remains an unacceptable burden on New Zealanders particularly those living in rural communities and on Māori. Famers and meat-workers remain most at risk and key intervention strategies for these occupations are the use of personal protective equipment (PPE) and animal vaccination. I will present our work on leptospirosis at the human-animal interface with focus on meat-workers who are required to use PPE yet have no say in the vaccination status of animals they are exposed to.

\section{K-04 CONCEPTUALIZING CHILD LABOR IN CONFLICT SETTINGS: THE CASE OF SYRIAN REFUGEES}

Rima Habib. American University of Beirut, Lebanon

\subsection{6/OEM-2019-EPI.4}

The persistence of child labor as a widespread global phenomenon is a result of the failure to challenge the conditions of economic injustice and child exploitation. Underlying this failure are policy frameworks that do not address the underlying conditions that produce child labor, namely state fragility, armed conflict, and environmental problems. An estimated 250 million children live in armed-conflict affected areas (Raqib, 2017), where the incidence of child labor is at least $77 \%$ higher than the global average (International Labour Organization, 2017). UNICEF (2016) estimates that about 535 million children live in conflict or disaster-stricken areas. Child labor thrives under these conditions because of the poverty, vulnerability, and lack of opportunities characteristic of areas affected by conflict and ecological disasters. The international system has not addressed the root causes of child labor, failing to raise and coordinate adequate relief and development resources when conflicts transpire.

This presentation will propose a new theoretical framework on child labor that incorporates the prominent role state fragility and armed conflict play in the constitution of this phenomenon. The presentation will further explore this theoretical framework through a case study of child labor in 